\title{
Synchronization of coupled single-electron circuits based on nanoparticles and tunneling junctions
}

\author{
Javier Cervera, José A. Manzanares, and Salvador Maféa) \\ Facultat de Física, Universitat de València, E-46100 Burjassot, Spain
}

(Received 4 December 2008; accepted 3 February 2009; published online 9 April 2009)

\begin{abstract}
We explore theoretically the synchronization properties of a device composed of coupled single-electron circuits whose building blocks are nanoparticles interconnected with tunneling junctions. Elementary nanoscillators can be achieved by a single-electron tunneling cell where the relaxation oscillation is induced by the tunneling. We develop a model to describe the synchronization of the nanoscillators and present sample calculations to demonstrate that the idea is feasible and could readily find applications. Instead of considering a particular system, we analyze the general properties of the device making use of an ideal model that emphasizes the essential characteristics of the concept. We define an order parameter for the system as a whole and demonstrate phase synchronization for sufficiently high values of the coupling resistance. (C) 2009 American Institute of Physics. [DOI: 10.1063/1.3095487]
\end{abstract}

\section{INTRODUCTION}

Synchronization is the adjustment of rhythms of oscillating objects because of their mutual interaction. ${ }^{1}$ It plays a central role in many biological (functioning of heart pacemaker cells and neurons in visual cortex), chemical (coupled chemical reactions and electrochemical oscillations), and applied physics (microelectromechanical resonators, microwave systems, and lasers) phenomena. ${ }^{1-6}$ In particular, synchronization circuits are fundamental to the correct behavior of many internal operations in electronic devices and digital circuitry.

Single-electron devices have attracted great interest as a future solution to the continuous miniaturization of electronics. ${ }^{7-9}$ These devices could form the basis of a new generation of processing information systems, although severe fabrication challenges have made practical implementations difficult. The experimental characterization and modeling of particular systems are receiving much attention at the elementary device level. However, it is also necessary to design general schemes that could perform information storage and processing tasks using well-defined nanostructures at the ideal circuit level..$^{7,8,10-12}$ We have recently proposed a device that uses nanoscale switches to implement a signal processing scheme and a frequency-dependent associative memory. ${ }^{13}$ In this article, we explore theoretically the synchronization of coupled single-electron circuits whose building blocks are nanoparticles interconnected with tunneling junctions.

Single-electron circuits may perform electronic functions on the basis of the transport of individual electrons, producing nonlinear oscillations and a rich dynamics. ${ }^{10,14,15}$ Elementary nanoscillators can be achieved by a singleelectron tunneling cell where the relaxation oscillation is induced by the tunneling. ${ }^{14-17}$ The tunnel junction acts as a leaky capacitor that can be controlled by the voltage across

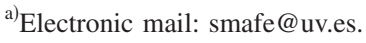

the junction and, because of the scale at which transport occurs, the transference of a few electrons may suffice to implement a variety of electronic functions. ${ }^{8}$ Electron tunneling is influenced by the discrete nature of charge transport and the Coulomb blockade energy barrier (this energy must be higher than the thermal energy for the transport of single electrons to be controlled). This condition is usually fulfilled by scaling down to molecular dimensions. The practical problems to overcome are the need to design the elementary building blocks at the nanometer scale ${ }^{12}$ and the random background charges that may affect reliable operation. ${ }^{10}$

\section{MODEL}

We develop a simple approach to describe the physical properties of the single-electron nanoscillators and present sample calculations to demonstrate that the idea is feasible and could readily find applications. Rather than considering a particular system, we analyze the general properties of the proposed device making use of an ideal model that emphasizes the essential characteristics of the concept. The building block of the device is composed of a nanoparticle (NP) of capacitance $C$ linked to an electrode (ground potential) by a tunneling junction (e.g., an organic ligand; ligandstabilized metallic nanoparticles ${ }^{18-21}$ permit tunable Coulomb blockade properties and single-electron tunneling ${ }^{13,22,23}$ ) and to a second electrode (applied potential $V_{0}$ ) by a high resistance $R$ [Fig. 1(a)]. Circuits with resistors connected to molecules (e.g., carbon nanotube transistors ${ }^{24}$ ) have experimentally been demonstrated, although the parasitic capacitance of the wires connected to the resistors may limit the operating frequency of the oscillations. ${ }^{24}$

At low temperatures, the Coulomb blockade effect causes the relaxation oscillation ${ }^{14,15}$ shown in Fig. 1(b). The potential $V$ of the nanoparticle increases through the charging process until it reaches the threshold value $e /(2 C)$, and then it decreases rapidly to $-e /(2 C)$ following the electron tunneling, from the ground to the nanoparticle, that occurs through the junction. The periodic sequence charging- 
(a)
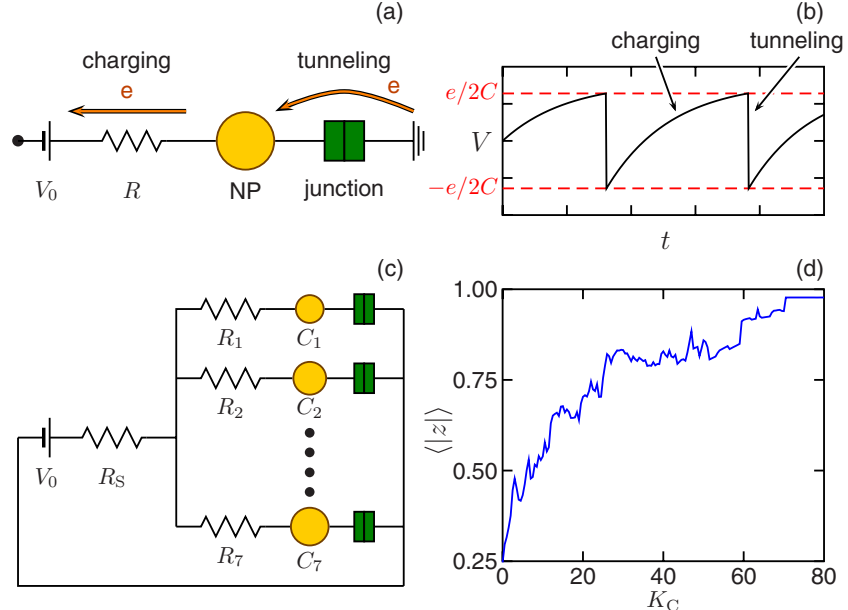

FIG. 1. (Color online) Scheme of the single-electron circuit (a) and the nanoparticle potential oscillation (b) due to the charging-tunneling processes. The proposed synchronization device is formed by seven elementary nanoscillators coupled through resistance $R_{S}(\mathrm{c})$. The time average value of the order parameter $z$ as a function of the coupling strength $K_{C}$ (d).

tunneling makes the potential of the nanoparticle oscillate [Fig. 1(b)]. The circuit dynamics ${ }^{14}$ can be approximately described by the differential equation

$$
C \frac{d V}{d t}=\frac{V_{0}-V}{R}
$$

for the voltage increase and the discrete equation

$$
\Delta V=-\frac{e}{C}, \quad V>\frac{e}{2 C}
$$

for the subsequent voltage drop, where $\Delta V$ is the potential difference before and after the electron tunneling. Because of the discreteness of the electron, the charging of the nanoparticle occurs in a multistep process. Nevertheless, it is modeled here using a continuum approach, since this approximation captures the basic characteristics of the process. ${ }^{14,15,25}$ The approach is valid when the number of elementary steps is sufficiently high so that the charging time is approximately constant. In addition, although there is a time lag (because of the stochastic nature of the tunneling process) between the instant when the threshold voltage is reached and the instant when tunneling occurs, this time is very small compared to the charging time for high values of the resistance $R \cdot{ }^{14}$ For the nanoscillator to have a definite frequency, the charging process needs to be rate limiting and determine the natural frequency of the oscillations. A method to define different frequencies is also needed and this can be accomplished using the values of the individual resistances and capacitances of the oscillators.

We consider now the device of Fig. 1(c) composed by seven nanoscillators similar to that of Fig. 1(a) that are coupled through the common resistance $R_{S}$, analyze the circuit dynamics, and describe the synchronization phenomena. The circuit variables are the nanoparticle potentials $V_{i}(i$ $=1,2, \ldots, 7)$. Because of the coupling resistance $R_{S}$, these potentials act as oscillators that synchronize for sufficiently high values of $R_{S}$. Synchronization occurs because when either of the potentials reaches the threshold value, tunneling proceeds through the corresponding junction, and this affects the other circuit branches. The nanoparticles act as capacitors whose capacitance is of the order of $1 \mathrm{aF}^{21,23}$ The charging rate depends on the applied potential $V_{0}$ and the individual resistances $R_{i}$,

$$
\sum_{j=1}^{N}\left(R_{i} \delta_{i j}+R_{S}\right) C_{j} \frac{d V_{j}}{d t}=V_{0}-V_{i},
$$

where $\delta_{i j}$ is the Kronecker delta. Equation (3) shows that the rate $d V_{i} / d t$ of each nanoparticle depends on the other potentials $V_{j}$ through the coupling resistance $R_{S}$. The coupling strength is characterized by the dimensionless parameter

$$
K_{C} \equiv \frac{R_{S}}{R_{0}} ; \quad \frac{1}{R_{0}}=\sum_{i=1}^{N} \frac{1}{R_{i}} .
$$

\section{RESULTS AND DISCUSSION}

To analyze the synchronization process, we define an effective individual period by calculating the time between two consecutive potential maxima, $T_{i}=t_{i+1}-t_{i}$ (this period is not, in general, strictly constant). This allows to define an individual phase $\theta_{i}(t) \equiv 2 \pi\left(t-t_{i}\right) / T_{i}$ for a time $t$ between $t_{i}$ and $t_{i+1} .{ }^{1}$ The level of synchronization of the system can then be described by the complex order parameter ${ }^{4,5}$

$$
z(t) \equiv \frac{1}{N} \sum_{j=1}^{N} e^{i \theta_{j}} .
$$

The nanoscillators of Fig. 1(c) generate the collective average signal shown in Fig. 1(d). In the incoherent regime (no coupling, $K_{C}=0$ ), the individual phases of the oscillators are uncorrelated and the time average of the modulus of $z$ is close to zero $(\langle|z|\rangle \rightarrow 0$ for a large number of oscillators). With the increase of the coupling parameter $K_{C},\langle|z|\rangle$ increases and, for sufficiently high values of $K_{C}$, full synchronization is achieved $(\langle|z|\rangle=1$ and the collective signal becomes trivially periodic). This behavior has recently been observed experimentally for the oscillating potentials in arrays of coupled microelectrodes. ${ }^{3-5}$ The results of Fig. 1(d) were obtained with equal resistances $R_{i}=R_{0}=10^{9} \Omega$ (this should be much higher than the tunneling resistance), different capacitances $C_{i}=i \mathrm{aF}, i=1,2, \ldots, 7$ [these can be achieved by varying the nanoparticle diameters ${ }^{19-21}$ in Fig. $1(\mathrm{c})$ ], and $V_{0}=100 \mathrm{mV}$. The coupling resistance $R_{S}$ can be obtained from $K_{C}$ in each case [Eq. (4)].

To better understand the synchronization achieved in Fig. 1(d), Fig. 2 shows the potentials of oscillators 1, 4, and 7 as a function of time for the coupling strengths $K_{c}=0$ (a), 10 (b), 40 (c), and 80 (d). Alternatively, Fig. 3 shows the trajectory of the order parameter $z(t)$ in the complex plane for the same coupling strengths of Fig. 2. Initially, the potentials $V_{j}$ of all oscillators increase with time $\left(V_{0}-V_{j}>0\right)$. The coupling resistance $R_{S}$ not only causes an increase in the nanoparticle charging times but also connects all oscillators through the potential $V_{S}$ because the charging current of oscillator $i$ is $I_{i}=\left(V_{S}-V_{i}\right) / R_{i}$, with $V_{S}=V_{0}-R_{S} \Sigma_{j=1}^{N} I_{j}$ [see Fig. $1(\mathrm{c})]$. The time variation of the potential $V_{i}$ is transmitted 


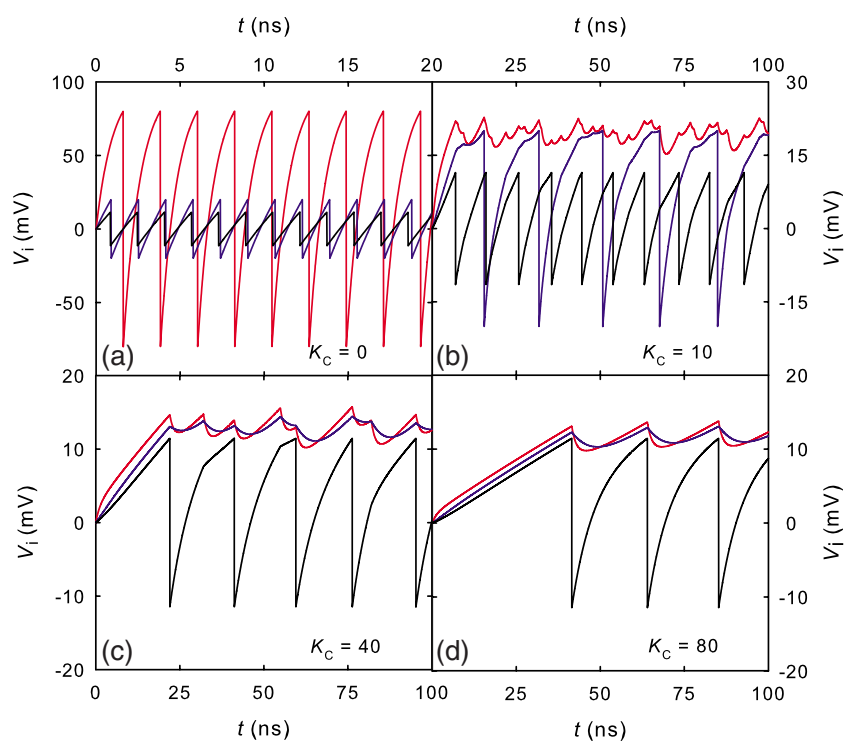

FIG. 2. (Color online) The nanoparticle potential of oscillators 1 (top curve), 4 (middle curve), and 7 (bottom curve) of Fig. 1(c) as a function of time for the coupling strengths $K_{C}=0$ (a), 10 (b), 40 (c), and 80 (d).

through $V_{S}$ to the currents $I_{j}(j \neq i)$ of the other oscillators and, finally, to the other potentials $V_{j}$. For instance, following the tunneling of an electron in oscillator $i$, the potential $V_{i}$ decreases by $\Delta V_{i}=-e / C_{i}$. As a consequence, an initial increase in the current $I_{i}=\left(V_{S}-V_{i}\right) / R_{i}$ (and then of the rate $\left.d V_{i} / d t\right)$ occurs [see Fig. 1(b)]. The increase in $I_{i}$ leads to a decrease of $V_{S}$, which in turn gives a decrease in $I_{j}(j \neq i)$ because of the coupling. This process eventually couples all rates $d V_{j} / d t$ for $K_{C} \neq 0$ [see Figs. 2(c) and 2(d)].

The progressive increase of $R_{S}$ in Figs. 2(a)-2(d) enhances the influence of the coupling term $R_{S} \Sigma_{j=1}^{N} I_{j}$ on $V_{S}$, making it more sensitive to the variation of any of the charging currents $I_{j}$. Because a change in $V_{S}$ affects all charging currents (and then all potentials $V_{i}$ ), the increase of $R_{S}$ eventually causes the collective synchronization of the oscillators

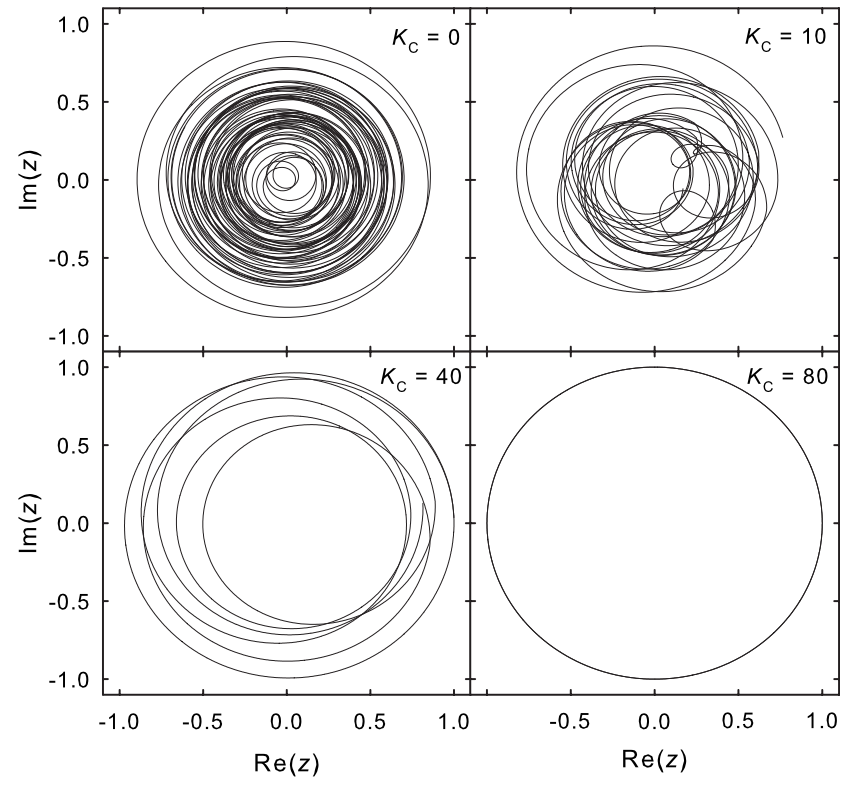

FIG. 3. The trajectory of the order parameter in the complex plane for the coupling strengths $K_{C}$ of Fig. 2.

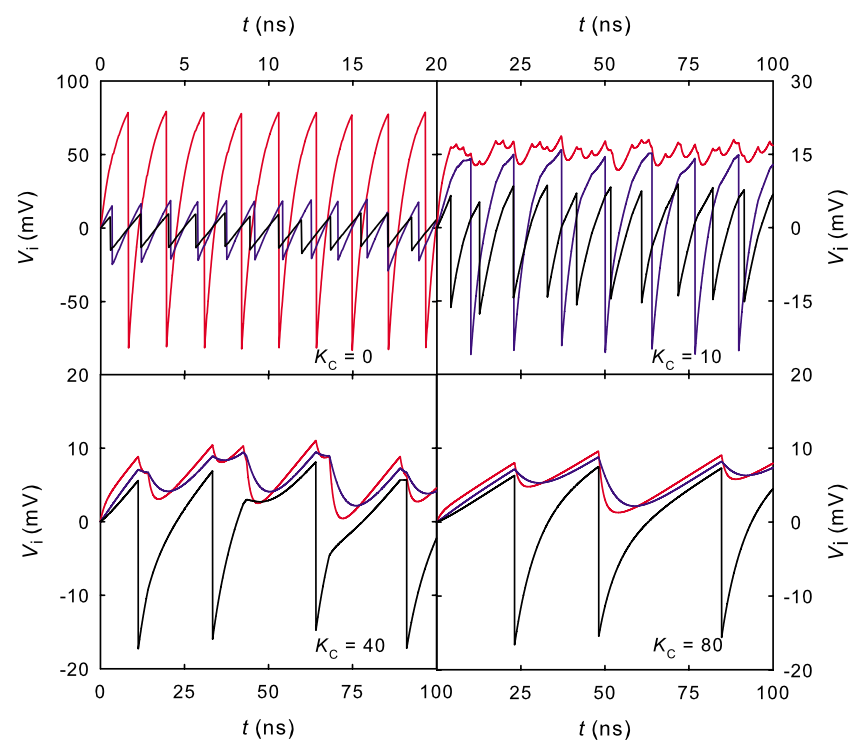

FIG. 4. (Color online) The nanoparticle potential of the oscillators of Fig. 2 for $T=10 \mathrm{~K}$ and the same parameters, with a tunneling resistance $R_{\text {tun }}$ $=10^{6} \Omega$.

[Fig. 2(d)]. Also, the equation $d V_{i} / d t=\left(V_{S}-V_{i}\right) /\left(R_{i} C_{i}\right)$ for nanoparticle $i$ shows that the dependence of the charging rate on the effective potential $V_{S}$ is high for those nanoparticles with low capacitances (compare $V_{1}$ with $V_{4}$ in Fig. 2). Therefore, switching to the effective synchronizing frequency is achieved first for the oscillators with low capacitances $\left(C_{1}\right.$ $<C_{2}<C_{3}$ ), the other becoming subsequently synchronized only with the increase in the values of $R_{S}$. For a sufficiently high $K_{C}$, all oscillators become synchronized and, as typical for relaxation oscillators, ${ }^{1}$ the oscillator with a shortest period (oscillator 7 here) sets the pace for the concerted potential variation [compare Fig. 2(a) with Fig. 2(d)] toward an effective synchronization frequency different from the natural ones (because of the coupling resistance $R_{S}$ ). Alternatively, the synchronization process of the seven oscillators can be visualized globally in Fig. 3. This figure shows the trajectory in the complex plane of the order parameter toward the limit circle of Fig. 3(d) (full synchronization), which is achieved for sufficiently high values of the coupling parameter $K_{C}$ [Fig. 1(d)].

The results of Fig. 2 concern single-electron nanoscillators at zero temperature. Preliminary Monte Carlo simulations at finite $T$ showed that, for the parameters used in Fig. $1(\mathrm{~d})$, synchronization is still possible at temperatures around $10 \mathrm{~K}$ (see Fig. 4). When the system is at a finite temperature, electron tunneling may occur at a voltage below $e / 2 C$ because of the thermal energy. If the tunneling resistance $R_{\text {tun }}$ is much higher than the quantum resistance $R_{h}=h / 2 e^{2}$ $=12.9 \mathrm{k} \Omega$, electron tunneling can be described by the rates ${ }^{13}$

$$
\Gamma_{\rightleftarrows}=\frac{1}{e^{2} R_{\text {tun }}} \frac{-\Delta E_{\rightleftarrows}}{1-\exp \left(\Delta E_{\rightleftarrows} / k T\right)},
$$

where the arrows indicate tunneling from the ground to the $\mathrm{NP}(\leftarrow)$ and from the NP to the ground $(\rightarrow)$, and $\Delta E$ is the increase of the electrostatic energy associated with electron tunneling ${ }^{13}$ 


$$
\Delta E_{\rightleftarrows}=\frac{e^{2}}{C}\left(\frac{1}{2} \pm \frac{C V}{e}\right) .
$$

To estimate the effect of temperature on the synchronization, we use a mixed continuum-Monte Carlo simulation approach. We employ a continuum approach for the NP charging and, at every charging step, we analyze if electron tunneling occurs by means of a Monte Carlo simulation. First, the values of $\Delta E_{\rightleftarrows}$ for each oscillator are calculated, and a Metropolis algorithm is used to decide which oscillators may undergo electron tunneling. Second, a kinetic Monte Carlo algorithm is employed to select the particular oscillator where electron tunneling occurs, assigning a time increase $\Delta t$ to the process. Figure 4 shows the results obtained for $T$ $=10 \mathrm{~K}$ using the parameters of Fig. 2, with a tunneling resistance $R_{\text {tun }}=10^{6} \Omega$ for all oscillators. At this temperature, the synchronization of the system is not significantly affected by the thermal energy. As expected, synchronization requires now relatively higher values of $K_{C}$. Also, the period of the synchronized oscillations shows small fluctuations (because of the stochastic nature of the tunneling process) around the average frequency of the synchronized signal.

\section{CONCLUSION}

In summary, we have designed a preliminary device that allows synchronizing a set of coupled single-electron circuits whose building blocks are nanoparticles interconnected with tunneling junctions (e.g., organic ligand-stabilized metallic nanoparticles), developed a simple model to describe the synchronization, and presented sample calculations to demonstrate the idea, emphasizing the essential characteristics that would be required for practical implementation.

\section{ACKNOWLEDGMENTS}

Financial support from the European Commission (project DYNAMO, Grant No. FP6-028669-2) under the program New Emerging Science and Technology is acknowledged.

${ }^{1}$ A. Pikovsky, M. Rosenblum, and J. Kurths, Synchronization: A Universal Concept in Nonlinear Science (Cambridge University Press, New York, 2001).

${ }^{2}$ S. H. Strogatz, Nonlinear Dynamics and Chaos: With Applications to Physics, Biology, Chemistry, and Engineering (Perseus, New York, 1994).

I. Z. Kiss, Y. Zhai, and J. L. Hudson, Science 296, 1676 (2002).

${ }^{4}$ A. S. Mikhailov, D. H. Zanette, Y. M. Zhai, I. Z. Kiss, and J. L. Hudson, Proc. Natl. Acad. Sci. U.S.A. 101, 10890 (2004).

${ }^{5}$ Y. Zhai, I. Z. Kiss, H. Daido, and J. L. Hudson, Physica D 205, 57 (2005).

${ }^{6}$ F. C. Hoppensteadt and E. M. Izhikevich, IEEE Trans. Circuits Syst., I: Fundam. Theory Appl. 48, 133 (2001).

${ }^{7}$ M. P. Blencowe, Contemp. Phys. 46, 249 (2005).

${ }^{8}$ A. van Roermund and J. Hoekstra, Int. J. Circuit Theory Appl. 28, 563 (2000).

${ }^{9}$ V. Ray, R. Subramanian, P. Bhadrachalam, L.-C. Ma, C.-U. Kim, and S. J. Koh, Nat. Nanotechnol. 3, 603 (2008).

${ }^{10}$ T. Yamanaka, T. Morie, M. Nagata, and A. Iwata, Nanotechnology 11, 154 (2000).

${ }^{11}$ N. Weibel, S. Grunder, and M. Mayor, Org. Biomol. Chem. 5, 2343 (2007).

${ }^{12}$ S. Mafé, J. A. Manzanares, and J. Cervera, J. Phys. Chem. C 112, 1663 (2008).

${ }^{13}$ J. Cervera, P. Ramírez, and S. Mafé, J. Appl. Phys. 104, 084317 (2008).

${ }^{14}$ A. K. Kikombo, T. Oya, T. Asai, and Y. Amemiya, Int. J. Bifurcation Chaos 17, 3613 (2007).

${ }^{15}$ T. Oya, T. Asai, and Y. Amemiya, Chaos, Solitons Fractals 32, 855 (2007).

${ }^{16}$ K. Likharev and A. B. Zorin, J. Low Temp. Phys. 59, 347 (1985).

${ }^{17}$ D. V. Averin and K. Likharev, J. Low Temp. Phys. 62, 345 (1986).

${ }^{18}$ M. Brust, M. Walker, D. Bethell, D. J. Schiffrin, and R. Whyman, J. Chem. Soc., Chem. Commun. 1994, 801.

${ }^{19}$ S. Chen, R. S. Ingram, M. J. Hostetler, J. J. Pietron, R. W. Murray, T. G. Schaaff, J. T. Khoury, M. M. Alvarez, and R. L. Whetten, Science 280, 2098 (1998).

${ }^{20}$ B. M. Quinn, P. Liljeroth, V. Ruiz, T. Laaksonen, and K. Kontturi, J. Am. Chem. Soc. 125, 6644 (2003).

${ }^{21}$ V. García-Morales and S. Mafé, J. Phys. Chem. C 111, 7242 (2007).

${ }^{22}$ B. Wang, H. Wang, H. Li, C. Zeng, J. G. Hou, and X. Xiao, Phys. Rev. B 63, 035403 (2000)

${ }^{23}$ J. Cervera and S. Mafé, Chem. Phys. Lett. 451, 257 (2008).

${ }^{24}$ A. Bachtold, P. Hadley, T. Nakinishi, and C. Dekker, Science 294, 1317 (2001).

${ }^{25}$ T. Oya, T. Asai, T. Fukui, and Y. Amemiya, Int. J. Unconv. Comput. 1, 177 (2005). 